\title{
Cancers de la thyroïde après Tchernobyl. Importance de la prophylaxie par le KI
}

\section{SCHLUMBERGER*}

(Manuscrit reçu le 6 avril 1994)

RÉSUMÉ Depuis 1990, plus de 400 cancers de la thyroïde ont été observés chez l'enfant dans le Sud de la Belarus et dans le Nord de I'Ukraine. L'importance de l'augmentation de l'incidence, l'agressivité de ces cancers et leur découverte fréquente à un stade métastatique rendent mineur le rôle du dépistage. Plusieurs arguments plaident en faveur de la responsabilité de l'accident de Tchernobyl : 1) l'importance de la contamination initiale ; 2) la distribution géographique, l'augmentation de l'incidence étant observée au niveau des régions les plus contaminées ; 3) la distribution temporelle, l'augmentation de l'incidence étant progressive depuis 1990. L'iode 131 est vraisemblablement l'agent responsable de cette augmentation. Ces données renforcent l'importance de la prophylaxie par l'iodure de potassium de l'irradiation de la thyroïde en cas d'accident de centrale nucléaire, notamment chez les enfants où elle est pratiquement sans danger.

ABSTRACT Since 1990, more than 400 thyroid cancers have been observed in children in southern Belarus and northern Ukraine. Screening is of minor significance on account of the highly increased incidence of these cancers, their agressivity and frequent diagnosis at a metastatic stage. Several arguments speak for the responsibility of the Chernobyl accident : 1) importance of the initial contamination ; 2) geographical distribution, the incidence being higher in the more severely contaminated areas ; 3) time distribution, the incidence increasing steadily since 1990. Iodine 131 is very likely the agent responsible for this increase. These data underline the significance of KI prophylaxis of thyroid irradiation in case of nuclear power plant accident, especially for children on whom it is practically without any danger.

\section{Les faits}

L'explosion du réacteur numéro $4 \mathrm{du}$ site nucléaire de Tchernobyl le 26 avril 1986 à 1 h 23, a libéré des quantités considérables d'isotopes radioactifs dont environ 50 millions de $\mathrm{Ci}(1850 \mathrm{PBq}$ ) d'iode 131 (période 8,02 j) ainsi que des isotopes à demi-vie brève tels que le tellure 132 (période 3,26 j) en équilibre avec son descendant l'iode 132 (période $2,3 \mathrm{~h}$ ), et l'iode 133 (période $20,8 \mathrm{~h}$ ). Le vent soufflant du sud vers le nord, le panache radioactif a atteint le Nord de l'Ukraine et le Sud de la Belarus et de la Russie. Dans ces régions, la contamination des sols par l'iode 131 a été au maximum de $1500 \mathrm{Ci} / \mathrm{km}^{-2}$

* Institut Gustave-Roussy, 94805 Villejuif Cedex, France. 
(555 $000 \mathrm{GBq} / \mathrm{km}^{-2}$ ) dans de nombreuses zones a été comprise entre 10 et $300 \mathrm{Ci} / \mathrm{km}^{-2}\left(370-11100 \mathrm{GBq} / \mathrm{km}^{-2}\right)$.

En cas de contamination par l'iode radioactif, la thyroïde est l'organe critique. Chez un adulte normal (fixation de la thyroïde à $24 \mathrm{~h}$ égale à $25 \%$ ), après l'administration d'1 $\mathrm{mCi}(37 \mathrm{MBq})$ d'iode 131 , la dose délivrée à la thyroïde est de $1300 \mathrm{cGy}$ [1] et la dose aux autres organes est 1000 à 10000 fois plus faible. La dose délivrée à la thyroïde peut être encore plus importante lorsque la fixation de l'iode est plus élevée (carence iodée, par exemple) et lorsque la thyroïde est plus petite (enfant).

Lors de l'accident, il existait dans ces régions une carence alimentaire en iode, car la supplémentation iodée avait été interrompue en 1981 pour des raisons économiques; la prophylaxie de l'irradiation de la thyroïde par l'iodure de potassium (KI) et par les restrictions alimentaires n'a été entreprise que de manière tardive ( 3 à 6 jours après l'accident) et très partielle. Ceci explique l'importance des doses délivrées à la thyrö̈de, notamment chez les enfants, qui ont été supérieures à $1000 \mathrm{cGy}$ chez $1 \%$ d'entre-eux et comprises entre 200 et 1000 cGy chez environ $10 \%$.

En Belarus, une augmentation considérable du nombre de cancers de la thyroïde traités chez l'enfant de moins de 15 ans a été constatée dès 1990 [1, 10-11]. Des données similaires ont été observées dans le Nord de l'Ukraine [16] (tableau I) et, plus récemment, dans la région de Bryansk, située au Sud de la Russie (G. Gerasimov, communication personnelle, mai 1994).

En dehors de ces régions, aucune augmentation de l'incidence des cancers de la thyroïde de l'enfant n'a été jusqu'à présent établie. Le recensement de ces cancers est facilité par le fait que ceux survenant dans le Nord de l'Ukraine sont traités à l'Institut d'endocrinologie de Kiev, et que ceux survenant en Belarus sont traités à Minsk. Un total de plus de 400 cancers thyroïdiens de l'enfant a donc été rapporté depuis 1989 en Belarus et en Ukraine. Ceci est à comparer avec les 106 cancers de la thyroïde observés à l'Institut Gustave Roussy (IGR) chez des enfants de moins de 17 ans pendant une période de 43 ans (entre 1950 et 1993), parmi les 2200 patients traités à l'IGR pour cette affection [19].

TABLEAU I

Cancers de la thyroïde chez les enfants âgés de moins de 15 ans lors du diagnostic $[10,16]$

Thyroid cancers in children under 15 at the time of the diagnosis $[10,16]$

\begin{tabular}{|lccccccc|}
\hline & $1986-88$ & 1989 & 1990 & 1991 & 1992 & 1993 \\
\hline Belarus & $2-5 /$ an & 7 & 29 & 59 & 66 & 79 \\
Ukraine & $4-9 /$ an & 21 & 38 & 40 & 70 & - \\
\hline
\end{tabular}


L'incidence des cancers de la thyroïde chez l'enfant [13] est de 0,04/100 000/an en Pologne, de 0,1/100 000/an dans les pays nordiques et de 0,3/100 000/an aux Etats-Unis d'Amérique, ce qui est en accord avec les incidences rapportées en Belarus et en Ukraine avant 1988. Depuis, cette incidence a été multipliée par un facteur 10 en Belarus et en Ukraine, et même plus dans certaines régions, dont les régions de Gomel et de Brest.

L'incidence spontanée des cancers de la thyroïde chez l'adulte est plus élevée. Son incidence en Belarus a augmenté de 202 cas en 1987 à 416 cas en 1992 et 512 en 1993 (E.P. Demidchik, communication personnelle, mai 1994). Ceci peut être lié, au moins en partie, à une amélioration du dépistage, mais peut également être un prélude à une augmentation beaucoup plus importante dans les années à venir.

A l'inverse, aucune augmentation significative de l'incidence d'autres pathologies malignes, notamment des leucémies, n'a été observée chez l'enfant.

Quatre vingt treize pour cent des enfants qui ont développé un cancer de la thyroïde avaient moins de 9 ans lors de l'accident [22], et plus de la moitié des cancers sont apparus chez des enfants âgés de moins de 10 ans lors du diagnostic. La majorité de ces cancers sont papillaires (93\% en Belarus). Plus des trois quarts mesurent plus d'1 cm, la majorité s'étendent aux tissus périthyroïdiens ou s'accompagnent de métastases ganglionnaires cervicales. Les métastases pulmonaires ont été mises en évidence chez $5 \%$ de ces enfants, mais ce pourcentage est sans doute sous-estimé du fait de l'absence de possibilité d'effectuer des scintigraphies du corps entier à l'iode 131 .

La majorité des cas rapportés (74 en Belarus et $62 \%$ en Ukraine) sont survenus chez des enfants vivant dans les régions les plus contaminées par les isotopes radioactifs de l'iode. Toutefois, il n'a pas été établi de relation entre la dosimétrie individuelle (mais qui n'est disponible que pour une minorité d'enfants) et la survenue d'un cancer de la thyroïde.

\section{Les questions}

\subsection{L'augmentation de l'incidence des cancers de la thyrö̈de chez l'enfant est-elle réelle?}

La réalité de l'incidence des cancers de la thyroïde chez l'enfant a été confirmée par un groupe d'histopathologistes suisses et britanniques $[1,4]$.

\subsection{Existe-t-il un biais permettant de l'expliquer?}

La relation entre l'accident de Tchernobyl et cette augmentation a été mise en doute en raison de deux arguments principaux : 
- l'administration d'iode 131 à visée médicale chez l'adulte (diagnostique et thérapeutique pour maladie de Basedow) n'a pas provoqué d'augmentation de l'incidence des cancers de la thyroïde, malgré un suivi prolongé $[7,8]$;

- l'irradiation externe chez l'enfant augmente l'incidence des tumeurs de la thyroïde, mais après une période de latence d'au moins 5 ans [20].

De ce fait, plusieurs points méritent d'être discutés :

\section{a) Le rôle du dépistage}

Des microcancers de la thyroïde, découverts lors d'autopsies systématiques, sont fréquents [6]; or, les méthodes de dépistage se sont considérablement améliorées depuis 1986, notamment grâce à l'introduction de l'échographie [2].

En fait, les cancers occultes sont fréquents chez l'adulte, mais rares chez l'enfant. Ainsi, une étude autopsique effectuée à Minsk a trouvé chez $10 \%$ des sujets âges de plus de 40 ans un microcancer papillaire, mais aucun chez ceux âges de moins de 40 ans [5]. Le dépistage des tumeurs de la thyroïde effectué chez les habitants de Chicago qui avaient été irradiés pendant l'enfance, a permis de découvrir un nombre important de tumeurs, mais celles-ci étaient de petites dimensions et sont survenues à un âge adulte [18].

A l'inverse, la plupart des tumeurs survenant chez les enfants d'Ukraine et de Belarus étaient volumineuses et s'accompagnaient d'extension ganglionnaire et/ou périthyroïdienne. L'histologie a montré de nombreux critères d'agressivité. Ces tumeurs sont ainsi comparables dans leur présentation clinique et histologique aux tumeurs spontanées du jeune enfant observées à l'IGR [19], dont l'évolution est souvent rapide en l'absence de traitement. Il est donc probable que le dépistage ait permis d'effectuer dans une minorité des cas, un diagnostic plus précoce de quelques mois, mais il ne peut expliquer cette augmentation considérable de l'incidence des cancers thyroïdiens chez l'enfant.

\section{b) La distribution géographique et temporelle}

L'analyse de cette distribution plaide en faveur de la responsabilité de l'accident de Tchernobyl :

- la majorité des cancers thyroïdiens sont survenus chez les enfants des régions les plus contaminées du Sud de la Belarus (notamment les régions de Gomel et de Brest) et du Nord de l'Ukraine, et, récemment, du Sud de la Russie ;

- la survenue très précoce des cancers de la thyroïde a été observée essentiellement chez des enfants qui étaient nouveau-nés ou encore in utero en 1986.

L'incidence de ces cancers continue d'augmenter, de $25 \%$ en Belarus, entre 1992 et 1993 [10]. 
Il faut, par ailleurs, souligner que quelques cas de cancers ont été décrits moins de 5 ans après une irradiation externe [20]. Dans ces études, l'incidence des tumeurs de la thyroïde a fortement augmenté entre 5 et 15 ans après l'irradiation. Ceci fait craindre des incidences encore supérieures aux incidences actuelles dans les années à venir.

La responsabilité de l'accident de Tchernobyl dans la survenue de ces cancers ne peut être établie de manière définitive que par des études épidémiologiques et dosimétriques rigoureuses, actuellement non disponibles. Il existe, toutefois, un ensemble d'arguments la suggérant très fortement, mais aucun argument permettant de la récuser.

\section{c) L'iode 131 est-il l'agent responsable?}

L'utilisation médicale de l'iode 131 n'a provoqué aucune augmentation ultérieure de l'incidence des cancers thyroïdiens $[7,8]$. Toutefois, les cohortes étudiées sont composées d'adultes et très peu de jeunes enfants ont été exposés à l'Iode 131 à visée médicale.

D'autres facteurs étiologiques ont donc été proposés : alimentaires (facteurs toxiques ou carentiels); pollution d'origine industrielle; éléments traces; carence iodée, mais celle-ci augmente essentiellement l'incidence des cancers vésiculaires, alors que la majorité des cancers observés étaient papillaires, comme ceux observés après irradiation externe ; contamination simultanée par les isotopes de l'iode à demi-vie brève et dont le débit de dose est élevé. Cette contamination a été rendue responsable de la survenue de cancers de la thyroïde après l'accident des Iles Marshall où elle a contribué aux $2 / 3$ de la dose totale délivrée à la thyroïde. En Ukraine et en Belarus, sa contribution à la dose totale délivrée à la thyroïde n'a pas excédé $10 \%$ [17].

Des études expérimentales chez le rat $[14,23]$ ont montré que l'iode 131 est cancérigène et que cet effet est augmenté par un traitement ultérieur par un goîtrigène (qui stimule la sécrétion de TSH et ainsi la prolifération cellulaire) et est diminué par l'hypophysectomie ou par un traitement prolongé par thyroxine (qui inhibe la sécrétion de $\mathrm{TSH}$ et ainsi la prolifération cellulaire). Ainsi, l'irradiation délivrée après une stimulation mitotique est moins cancérigène que celle délivrée avant une telle stimulation. Ceci suggère que la croissance cellulaire après l'irradiation a un effet très important dans la cancérogenèse thyroïdienne radioinduite.

En cas de maladie de Basedow, les cellules sont incapables de proliférer après le traitement par l'iode 131, comme en témoigne la grande fréquence de l'hypothyroïdie après un tel traitement. A l'inverse, la thyroïde de l'enfant, et surtout celle du jeune enfant et du fœtus, a des capacités prolifératives importantes. L'irradiation de ces cellules par l'iode 131 à un âge jeune peut donc avoir un effet cancérigène qui sera révélé au cours des divisions cellulaires ultérieures [21]. D'ailleurs, l'irradiation externe est beaucoup plus cancérigène chez l'enfant que chez l'adulte [20]. 
La nature des anomalies géniques provoquées par ces irradiations reste inconnue. Elles méritent d'être recherchées et comparées à celles existant dans les tumeurs de l'enfant, survenues de manière spontanée ou après irradiation externe.

En conclusion, ces études montrent que ces cancers de la thyroïde chez le jeune enfant sont attribuables à l'accident de Tchernobyl et sont probablement liés à l'irradiation de la thyroïde par l'iode radioactif. A l'inverse, aucune augmentation de l'incidence d'autres cancers ou de leucémies n'a été observée depuis 1986. En cas d'accident de centrale nucléaire, l'irradiation de la thyroïde par l'iode radioactif doit donc être prévenue, notamment chez l'enfant, ce qui peut être réalisé par la prise d'iodure de potassium associée à des restrictions alimentaires.

\section{Prophylaxie par l'iodure de potassium}

En cas de contamination par l'iode radioactif, la prise d'iodure de potassium (KI) peut prévenir l'irradiation de la thyroïde, à trois conditions :

- prise d'une quantité suffisante de $\mathrm{KI}$,

- adulte : $100 \mathrm{mg}$ d'iodure (y compris les femmes enceintes),

- enfant $<13$ ans : $50 \mathrm{mg}$ d'iodure,

- enfant $<3$ ans : $25 \mathrm{mg}$ d'iodure ;

ces quantités sont celles recommandées par la circulaire du ministère de l'intérieur et du ministère de la santé du 18 août 1992 [3], les comprimés actuellement disponibles en France contenant $130 \mathrm{mg}$ de KI, soit $100 \mathrm{mg}$ d'iodure ;

- prise rapide après la contamination (voire quelques heures avant celle-ci),

- prise répétée en cas de contamination prolongée.

Si ces conditions sont respectées, l'irradiation de la thyroïde est diminuée de plus de $98 \%$. Des restrictions alimentaires doivent être associées, concernant notamment le lait frais, particulièrement s'il n'est soumis à aucun contrôle.

L'efficacité de la distribution de KI à l'échelle d'un pays a été démontrée en Pologne où 18 millions de doses ont été distribuées (dont 10 millions chez les enfants). Ceci a permis de diminuer l'irradiation de la thyroïde à des niveaux considérés comme non dangereux [15]. De plus, la distribution du KI en Pologne en a démontré l'innocuité : aucune augmentation de l'incidence de l'hypothyroïdie à la naissance n'a été mise en évidence chez les nouveaux nés dont les mères avaient pris le KI pendant leur grossesse; une hypothyroïdie biologique a été observée chez $0,4 \%$ des nouveaux nés qui avaient absorbé le KI quelques jours après la naissance, mais celle-ci a été transitoire et a régressé sans aucune séquelle; deux accidents d'allergie ont nécessité une hospitalisation (sur 18 millions de personnes traitées) et $4 \%$ des personnes traitées ont eu 
des effets secondaires à type de troubles digestifs ou de rashs cutanés. La majorité a été sans gravité, et $5 \%$ de ces effets secondaires ont nécessité des soins ambulatoires.

Une prophylaxie par KI devra être instaurée lorsqu'elle permettra d'éviter à la thyroïde de recevoir une dose supérieure à $50 \mathrm{cGy}$. Cette prophylaxie peut être instaurée dans un contexte d'urgence pour des doses évitées moins importantes, mais la valeur de $50 \mathrm{cGy}$ ne doit pas être diminuée de plus d'un facteur 10 [9]. Rappelons que les enfants doivent recevoir en priorité cette prophylaxie par KI car, pour une contamination donnée, la dose délivrée à la thyroïde est d'autant plus élevée que la thyroïde est petite (donc que l'enfant est plus jeune); la thyroïde des enfants est plus sensible à l'action cancérigène des rayonnements que celle des adultes [20]; les incidents et accidents liés à la prise de KI sont exceptionnels chez l'enfant. De même, il est indispensable d'effectuer cette prophylaxie par KI chez les femmes enceintes afin d'éviter l'irradiation de la thyroïde fotale.

L'accident de Tchernobyl, a montré que les populations vivant à plusieurs centaines de kilomètres de la centrale (région de Brest notamment) peuvent être fortement contaminées et développer dans les années qui suivent un cancer de la thyroïde. Ceci montre que les plans d'intervention doivent être établis au niveau d'un pays, voire d'un continent.

\section{RÉFÉRENCES}

[1] BAVERSTOCK K., EGLOFF B., PINCHERA A., RUCHTI C., WILliAMS D. Thyroid cancer after Chernobyl. Nature, 1992, 359, 21-22.

[2] BERAL V., REEVES G. - Thyroid cancer after Chernobyl. Nature, 1992, 359, 680681.

[3] FRANCE. Ministère de l'intérieur et ministère de la santé. Circulaire DGS/92-45 relative à l'administration d'iode stable en cas d'accident nucléaire du 18 aout 1992.

[4] FURMANCHUK A.W., AVERKIN J.I., EGLOFF B., RUCHTI C., ABELIN T., SCHAPPI W., KOROTKEVICH E.A. - Pathomorphological findings in thyroid cancers of children from the Republic of Belarus : a study of 86 cases occurring between 1986 ("post-Chernobyl") and 1991. Histopathology, 1992, 21, 401-408.

[5] FURMANCHUK A.W., ROUSSAK N., RUCHTI C. - Occult thyroid carcinomas in the region of Minsk, Belarus, an autopsy study of 215 patients. Histopathology, 1993, $23,319-325$.

[6] HARACH H.R., FRANSSILA K.O., WASENIUS V.M. - Occult papillary carcinoma of the thyroid, a normal finding in Finland : a systematic autopsy study. Cancer, 1985, 56, 531-538.

[7] HOLM L.E., DAHLQUIST I., ISRAELSSON A., LUNDELL G. - Malignant thyroid tumors after iodine 131 therapy, a retrospective cohort study. New Engl. J. Med., 1980, 303, 188-191.

[8] HOLM L.E., WIKLUND K.E., LUNDELL G.E., BERMAN N.A., BJELKENGREN G., CEDERQUIST E.S., ERICSSON U.B., LARSSON L.G., LID- 
BERG M.E., LINDBERG R.S., WICKLUND H.V., BOICE J.D. - Thyroid cancer after diagnostic doses of iodine 131 : a retrospective cohort study. J. Natl. Cancer Inst, 1988, 80, 1132-1138.

[9] INTERNATIONAL COMMISSION ON RADIOLOGICAL PROTECTION (ICRP). - Principles for intervention for protection of the public in a radiological emergency (ICRP Publication 63). Oxford : Pergamon press, 1991.

[10] KAPLIYEVA M.P, ASTAKHOVA L.N. - Incidence of thyroid cancer of the children in Belarus after Chernobyl accident. Nagasaki symposium on Chernobyl, Nagasaki, 12 December, 1993.

[11] KAZAKOV V.S, DEMIDCHIK E.P, ASTAKHOVA L.N. - Thyroid cancer after Chernobyl. Nature, 1992, 359, 21.

[12] LOEVINGER R., BUDINGER T. F., WATSON E.E. - MIRD primer for absorbed dose calculations. New-York : The Society of nuclear medicine, 1988.

[13] MUIR C., WATERHOUSE J., MACK T., POWELL J., WHELAN S. - Cancer incidence in five continents. (IARC Scientific publications $n^{\circ} 88$ ) Lyon : IARC, 1987.

[14] NADLER N.J., MANDAVIA M., GOLDBERG M. - The effect of hypophysectomy on the experimental production of thyroid neoplasms. Cancer Res, 1970, 30, 1909-1911.

[15] NAUMAN J., WOLFF J. - Iodide prophylaxis in Poland after the Chernobyl reactor accident : benefits and risks. Am. J. Med, 1993, 94, 524-532.

[16] OLEYNIK V.A. - Thyroid cancer in children of Ukraine before and after Chernobyl accident. Nagasaki symposium on Chernobyl, Nagasaki, 12 December, 1993.

[17] PARMENTIER C., ROBEAU D., SCHLUMBERGER M., AUBERT B., NENOT J.C., PARMENTIER N. - L'après-Tchernobyl : des cancers en excès. La Recherche, 1993, 24, 738-740.

[18] RON E., LUBIN J., SCHNEIDER A.B. - Thyroid cancer incidence. Nature, 1992, $360,113$.

[19] SCHLUMBERGER M., de VATHAIRE F., TRAVAGLI J.P., VASSAL G., LEMERLE J., PARMENTIER C., TUBIANA M. - Differentiated thyroid carcinoma in childhood : long term follow-up of 72 patients. J. Clin. Endocrinol. Metab, 1987, 65, 1088-1094.

[20] SHORE R.E. - Issues and epidemiological evidence regarding radiation induced thyroid cancer. Radiat. Res., 1992, 131, 98-111.

[21] WILLIAMS E.D. - Radiation induced thyroid cancer. Histopathology, 1993, 23, 387-389.

[22] Williams E.D., PINCHera A., Karaoglou A., CHAdWICK K.H. Thyroid cancer in children living near Chernobyl. Rapport EUR-15248, 1993.

[23] WYNFORD-THOMAS D., STRINGER B.M.J., WILLIAMS E.D. - Dissociation of growth and function in the rat thyroid during prolonged goitrigen administration. Acta Endocrinol, 1982, 101, 210-216. 